\title{
Decaying Asymmetric Dark Matter Relaxes the AMS-Fermi Tension
}

\author{
Lei Feng ${ }^{1}$ and Zhaofeng Kang $^{2, *}$ \\ ${ }^{1}$ Key Laboratory of Dark Matter and Space Astronomy, \\ Purple Mountain Observatory, Chinese Academy of Sciences, Nanjing 210008, China \\ ${ }^{2}$ Center for High-Energy Physics, Peking University, Beijing, 100871, P. R. China
}

(Dated: June 21, 2018)

\begin{abstract}
The first result of AMS-02 confirms the positron fraction excess observed by PAMELA, but the spectrum is somewhat softer than that of PAMELA. In the dark matter (DM) interpretation it brings a tension between AMS-02 and Fermi-LAT, which reported an excess of the electron plus positron flux. In this work we point out that the asymmetric cosmic ray from asymmetric dark matter (ADM) decay relaxes the tension. It is found that in the case of two-body decay a bosonic ADM around $2.4 \mathrm{TeV}$ and decaying into $\mu^{-} \tau^{+}$can significantly improve the fits. Based on the $R$-parity-violating supersymmetry with operators $L L E^{c}$, we propose a minimal model to realize that ADM. The model introduces only a pair of singlets $(X, \bar{X})$ with a tiny coupling $L H_{u} X$, which makes the ADM share the lepton asymmetry and decay into $\mu^{-} \tau^{+}$along the operator $L L E^{c}$.

PACS numbers: 12.60.Jv, 14.70.Pw, 95.35.+d
\end{abstract}

*Electronic address: zhaofengkang@gmail.com 


\section{INTRODUCTION AND MOTIVATIONS}

Dark matter (DM) is commonly accepted as a major component of our Universe in the present era, and its fraction in the total energy budget is precisely determined to be $26 \%$ [1]. Nevertheless, the most confirmative evidences for its existence come from its gravitational effects, which renders its particle properties barely known. In the galaxy, DM may annihilate or decay into the cosmic ray (CR) components like $e^{ \pm}$and $p / \bar{p}$, which induces the CR excesses (or CR anomalies). Observations of such anomalies by means of indirect DM detections can be regarded as a smoking-gun for DM, and they may convey important information about the particle properties of DM.

Recently, such smoking-guns have been triggered. In the year 2008, PAMELA reported that, within the region from $10 \mathrm{GeV}$ to $100 \mathrm{GeV}$ [2], the positron fraction of CR shows a sharp rising excess over the background. This excess was extended to the higher energy by PAEMLA and FERMI [3], in spite of a larger error bar. It is of great interest to attribute the excess to extra $e^{+} / e^{-}$from DM decay or annihilation. More interestingly, this is well consistent with another important excess from the 2009 Fermi-LAT, which precisely measured the total electron plus positron flux and observed a flat excess up to the TeV region [4] [44]. On the other hand, both the PAMELA anti-proton [5] and Fermi-LAT diffuse gamma ray [6-8] data are well fitted by the pure backgrounds, which then stringently restrict the possible dark matter explanations. In practice, the inverse Campton scattering (ICS) process of the injected $e^{+} / e^{-}$always produces an associated diffuse gamma ray spectrum. In the annihilating and decaying DM scenarios, the injected $e^{+} / e^{-}$fluxes are respectively proportion to $\rho(r)^{2}$ and $\rho(r)$ with $\rho(r)$ the DM distribution function, so the decaying DM scenario is strongly favored [7] to evade the exclusion from Fermi-LAT. In supersymmetric standard models (SSMs), the required extremely long lifetime $\sim 10^{26}$ s and leptonic final states, can be realized by introducing proper $R$-parity violating operators [10], or dimension-six operators suppressed by the GUT-scale [11, 12] and further aided by some flavor symmetry [12].

Very recently, the long-expected AMS-02 experiment released the first result of positron fraction measurement [13]. It measures the fraction over a much wider energy region, from 0.5 to $350 \mathrm{GeV}$, with an unprecedented high precision. It confirms the excess, and largely speaking, is consistent with the result of PAMELA. However, at the same time it brings a clear tension with the Fermi-LAT total flux measurement [14-17, 19]. The tension is ascribed to the fact that the spectrum of AMS-02 is relatively softer than that of PAMELA, especially in the higher energy region (As stressed by Ref. [13], the slope of the spectrum decreases one magnitude of order from 10 to $250 \mathrm{GeV}$.). As a consequence, as we fit FermiLAT using a relatively heavy DM, the resulted positron fraction in the higher energy region will be too hard to fit AMS-02 (The same problem may also be encountered in the pul- 
sar explanation $[18,19])$. To reconcile AMS-02 and Fermi-LAT, we may need to consider nonconventional scenarios of $\mathrm{CR}$, which can give a relatively harder $e^{-}$spectrum than the $e^{+}$spectrum. This is explored in the astrophysical context like hardening the primary $e^{-}$ spectrum in the higher energy region $[14,19,20]$.

Viewing from particle physics, hardening the $e^{-}$spectrum can be naturally achieved by updating the decaying DM to the asymmetric decaying DM. In this framework, when the asymmetric DM decay produces asymmetric final states, says $\mu^{-} \tau^{+}$without the corresponding conjugate state $\mu^{+} \tau^{-}$, then the asymmetric cosmic ray with a harder $e^{-}$spectrum (than the $e^{+}$spectrum) is produced. Actually, such a scenario has already been studied even at the time when only the PAMELA data is available [21, 22]. AMS-02 result may favor it [45].

This paper is organized as follows. In Section II, a model independent fit of the AMS-02 and Fermi-LAT data is employed, assuming that the (scalar) asymmetric DM asymmetrically decays into a pair of charged leptons. In Section III, we propose a minimal supersymmetric decaying asymmetric DM model to implement the idea. The Section IV is the discussion and conclusion. A model for $R$-parity violation is given in Appendices A.

\section{ASYMMETRIC COSMIC RAY RELAXES THE AMS-FERMI TENSION}

Recently, the AMS-02 collaboration reported the first result, which confirms the excess of positron fraction observed several years ago by PAMELA. But the measured spectrum is mildly softer than that of PAMELA, as causes the difficulty in fitting AMS-02 and FermiLAT simultaneously. A way out of this difficulty is going beyond the symmetric CR and assuming that in the injected $\mathrm{CR}$ the $e^{-}$fraction is harder than the $e^{+}$fraction, namely

$$
r^{+}(E) \equiv \frac{\Phi^{+}(E)}{\Phi^{+}(E)+\Phi^{-}(E)}>0.5,
$$

with $\Phi^{ \pm}(E)$ the flux of $e^{ \pm}$, respectively. Note that such a solution thanks to the fact that Fermi-LAT is bind to the sign of charges. Asymmetric DM decay potentially produces such kind of asymmetry, and in this work we consider the following three asymmetric modes from a scalar DM $X$ decay (See also [21]):

$$
X \rightarrow e^{-} \mu^{+}, \quad e^{-} \tau^{+}, \quad \mu^{-} \tau^{+} .
$$

Two comments are in orders. First, if this asymmetry is generated by asymmetric DM decay, the case in this work, generically $r^{+}(E)$ has an energy dependence. Second, in Ref. [22] the case of a fermionic DM decaying into a charged lepton plus a charged Higgs boson or $W$ boson is considered, but it produces many anti-protons and thus is disfavored. Moreover, although not shown explicitly, the fits in this case becomes much worse. 

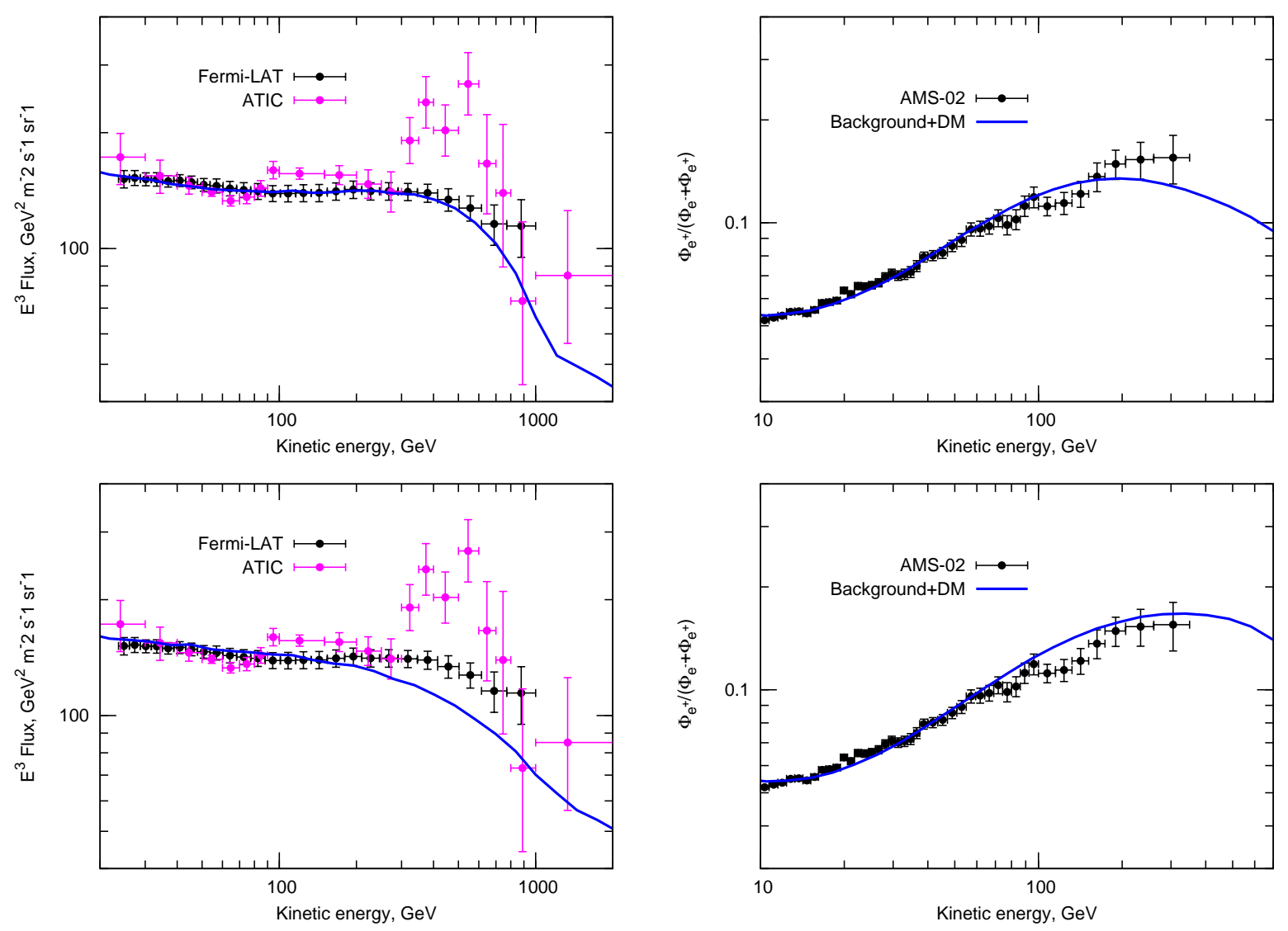

FIG. 1: Top-panels: Fits for the AMS-02 positron fraction spectrum (right) and the Fermi-LAT $e^{-}+e^{+}$flux spectrum (left), using an asymmetric cosmic ray originating in an asymmetric dark matter decaying to $\mu^{-}+\tau^{+}$. The best fit is achieved for the dark matter with mass $2.39 \mathrm{TeV}$ and lifetime $2.23 \times 10^{26} \mathrm{~s}$. Bottom-panels: A comparison to the best fit using the symmetric cosmic ray from DM decaying to $\tau^{+} \tau^{-}$.

Before heading towards data fitting, we introduce the astrophysical conventions/notations used in this paper. The propagation of CR in the Milk Way is described by a Boltzmann equation. For a given component of $\mathrm{CR}$, we can obtain its flux by solving this equation using the corresponding boundary condition. However, owing to the complicated distributions of sources, interstellar matter, radiation field and magnetic field, it is difficult to solve it analytically. Thus here we turn to the numerical code, the GALPROP package [23], and we modify the code to include the contribution from DM decay. In the fitting process, we take the following parameters for the CR propagation: The diffusion coefficient $D_{0}=$ $5.3 \times 10^{28} \mathrm{~cm}^{2} \mathrm{~s}^{-1}$, the diffusion index $\delta=0.33$, the Alfven velocity $V_{\mathrm{A}}=33.5 \mathrm{~km} \mathrm{~s}^{-1}$ and the Halo height $z_{\mathrm{h}}=4 \mathrm{kpc}$. Furthermore, the injection indexes of nucleon below and above the break rigidity $\rho_{\mathrm{br}}=11.5 \mathrm{GV}$ are 1.88 and 2.39, respectively. Finally, as for the DM 
distribution function, we use the Einasto density profile [24]:

$$
\rho(r)=\rho_{s} \exp \left(-\frac{2}{\alpha_{s}}\left[\left(\frac{r}{r_{s}}\right)^{\alpha_{s}}-1\right]\right),
$$

where $\alpha_{s}=0.17, \rho_{s} \approx 0.14 \mathrm{GeV} \mathrm{cm}^{-3}$ and $r_{s} \approx 15.7 \mathrm{kpc}$.

With our code based on the CosmoMC package [25], we now perform the Markov Chain Monte Carlo (MCMC) global fitting to determine the relevant parameters. We find that, among the three modes listed in Eq. (2), the best mode is $X \rightarrow \mu^{-} \tau^{+}$, with a $\chi^{2}=60.26$ and the degrees of freedom 63. The corresponding ADM mass and lifetime are $2.39 \mathrm{TeV}$ and $2.23 \times 10^{26} \mathrm{~s}$, respectively. The fits for the AMS-02 and Fermi-LAT data are shown in the top panels of Fig. 1. As a comparison, we also display the fits in the symmetric decaying DM case, see the bottom panels of Fig. 1. The best fit is achieved for a similar DM, with mass and lifetime $2.70 \mathrm{TeV}$ and $1.93 \times 10^{26} \mathrm{~s}$, respectively, but decaying into $\tau^{+} \tau^{-}$. The chi square is much worse, $\chi^{2}=142.45$ with the degrees of freedom 63 .

\section{ASYMMETRIC COSMIC RAY FROM ASYMMETRIC DM DECAY}

In particle physics, it is natural to expect that the asymmetric CR originates from asymmetric dark matter (ADM) decay [21, 22]. However, for the sake of naturally solving the cosmic coincidence puzzle, namely $\Omega_{b} h^{2}: \Omega_{\mathrm{DM}} h^{2} \simeq 6: 1$, the ADM mass is expected to be around $10 \mathrm{GeV}[22]$ rather than at the TeV scale inspired by solving the AMS-Fermi tension. Additionally, it is difficult to embed ADM into the simple supersymmetric standard models (SSMs) such as the minimal-SSM (MSSM) and its singlet (e.g., the NMSSM) or right-handed neutrinos extensions, owing to the robust neutralino mediated charge wash-out effect [27]. In this section we will first construct a simple and natural model to address these problems and then discuss the phenomenological aspects of the model.

\section{A. TeV-scale decaying ADM}

Within the conventional ADM framework [28], dark matter carries a generalized lepton or baryon number through proper couplings to the standard model (SM) matters. Effectively, this can be described by the operators such as $\mathcal{O}_{T}=\mathcal{O}_{\mathrm{DM}} \mathcal{O}_{\mathrm{SM}}(\ell, q)$, proposed by Ref. [36]. Above some temperature $T_{D}$, they establish the chemical equilibrium between the dark sector and visible sector and lead to [29]

$$
\sum_{i} \mu_{\phi_{i}}=0
$$


with $\mu_{\phi_{i}}$ the chemical potentials [29] of the particles appearing in the previous operators. In other words, they transfer the visible sector matter asymmetry (assumed to be generated via some visible sector dynamics) to the hidden sector, which is symmetric if $\mathcal{O}_{T}$ is turned off. Eq. (4) indicates that the chemical potentials of two sectors should be at the same order.

Further, for the particles in the thermal bath with temperature $T$, their asymmetries can be expressed with the corresponding chemical potentials [30]

$$
\begin{aligned}
n_{+}-n_{-} & =g \frac{T^{3}}{\pi^{2}} \frac{\mu}{T} \int_{0}^{\infty} d x \frac{x^{2} \exp \left[-\sqrt{x^{2}+(m / T)^{2}}\right]}{\left(\theta+\exp \left[-\sqrt{x^{2}+(m / T)^{2}}\right]\right)^{2}} \\
& \equiv \begin{cases}f_{b}(m / T) \times g_{b} \frac{T^{3}}{6}\left(\frac{\mu}{T}\right), & \text { (for bosons) } \\
f_{f}(m / T) \times g_{f} \frac{T^{3}}{6}\left(\frac{\mu}{T}\right), & \text { (for fermions) }\end{cases}
\end{aligned}
$$

where $\theta$ takes 1 and -1 for a boson and fermion respectively. $g$ denotes the internal degrees of freedom. The Boltzmann suppression factors $f_{b, f}(m / T)$ indicate the threshold effects for heavy particles. For particles in the ultra-relativistic limit, i.e., $m \ll T, f_{b, f}$ tend to 2 and 1 , respectively. In the opposite, the asymmetry of the decoupling particle can be greatly suppressed. Specified to DM and assuming that the symmetric part of its number density annihilates away, then the relic densities of baryonic and dark matters take the ratio:

$$
\frac{\Omega_{b} h^{2}}{\Omega_{X} h^{2}}=\frac{m_{p}}{m_{X}} \frac{\sum_{q} \mu_{q}}{g_{X} f_{X} \mu_{X}},
$$

with $m_{X}$ the DM mass. Thereby, when the transfer ceases at a temperature far above $m_{X}$, just the case for the conventional scenario [28], it is found that $m_{X} \sim 10 \mathrm{GeV}$ is predicted to solve the cosmic coincidence puzzle. By contrast, when the chemical equilibrium decoupling happens during the period of DM entering non-relativistic, then the puzzle has to be resolved by a heavy DM $[31,32]$. In spite of a sensitive dependence on the ratio $m / T$, ADM in this scenario still has a dynamical origin for its similar relic density to the baryonic matter's (Beyond the chemical equilibrium mechanism to generate DM asymmetry, there are other ways to get a heavy ADM [35].).

To realize a leptonic decaying ADM, it is tempting to consider that $\mathcal{O}_{T}$ not only transfers asymmetry but also provides a path for DM decaying into leptons. It can be achieved by assuming that $\mathcal{O}_{T}$ involve leptons only and $X$ develops a vacuum expectation value (VEV) $v_{X}$, which breaks the symmetry protecting DM stabile. However, bare in mind that here the decaying DM is extremely long lived, so either a very small $v_{X}$ or exceedingly suppression from the operator coefficients is indispensable. The former, in principle is possible given some complicated dynamics, while the latter is inconsistent with the TeV scale ADM set up because it requires the operators to decouple below $m_{X}$. Then we are led to the scenario where an extra source slightly violates the symmetry protecting DM stability. A good case 
in point is the $R$-parity-violating SUSY, where the tiny $R$-parity violations are spectator to the usual dark matter dynamics and only account for DM late decay. In the following subsection, based on the $R$-parity-violating SUSY we construct the minimal model for leptonic decaying ADM at the TeV scale.

\section{B. A minimal supersymmetric model}

Now we embed a heavy decaying ADM in SUSY. Due to the neutralino mediated washout effect [27], ADM is difficult to be accommodated in the popular supersymmetric models. Within the chemical equilibrium framework, Ref. [31, 32, 36] utilized high dimension operators like $\mathcal{O}_{T}=X^{2} L L E^{c}$ with a new and relatively low cut-off scale, to transfer asymmetry. Here we construct a more economic model, which even does not incur any new scales in the superpotential. The model takes a form of (Actually, it resembles the supersymmetric inverse seesaw model proposed in Ref. [37], where a light ADM is studied):

$$
\begin{aligned}
W & =\lambda_{i j k} L_{i} L_{j} E_{k}^{c}+\left(y_{i X} L_{i} H_{u} X+\lambda_{X} S X \bar{X}\right) \\
& +\left(\lambda S H_{u} H_{d}+\frac{\kappa}{3} S^{3}\right)+W_{\text {MSSM }} .
\end{aligned}
$$

The corresponding soft terms are implied. $L_{i}$ and $L_{j}$ are asymmetric, and thus $\lambda_{i j k}=-\lambda_{j i k}$. We order $i<j$ hereafter. The superpotential is divided into three sectors: the $R$-parityviolating sector which accounts for the ADM leptonic decay, the dark sector which transfers asymmetry between (s)leptons and DM, and the ordinary NMSSM sector, i.e., terms in the second line, which dynamically generates the low energy scales via the singlet $\operatorname{VEV}\langle S\rangle \equiv v_{s}$. In this model the dark sector fields $(X, \bar{X})$ respectively carry lepton number -1 and +1 , by virtue of the renormalizable transferring operator $\mathcal{O}_{T}=y_{i X} L_{i} H_{u} X$ (A single family will be considered for simplicity and $y_{i X} \rightarrow y_{X}$ hereafter). But the lepton number is explicitly violated by the first sector, so it fails to guarantee the general structure of the model. We may have to turn to other symmetries, such as the generalized $Z_{3}$ symmetry of the NMSSM. We will specifically address this problem somewhere else.

We now discuss several evident features of the model's parameter space. First, a large $\lambda_{X}$ is favored to annihilate away the symmetric part of DM number density. But we require the model to stay perturbative up to the GUT scale, so $\lambda_{X} \lesssim 0.5$ and then a sufficiently large singlet VEV, says $v_{s} \sim \mathcal{O}(5) \mathrm{TeV}$, is necessary to make $M_{X}=\lambda_{X} v_{s} \sim \mathcal{O}(\mathrm{TeV})$. To that end, a large $\lambda A_{\lambda}$ may be required in the NMSSM Higgs sector. Next, the soft spectrum is rather heavy because the $\mathrm{TeV}$-scale $\mathrm{ADM}$ is also the lightest sparticle (LSP). As a consequence, the Higgsino and singlino masses $\mu=\lambda v_{s}$ and $M_{\widetilde{s}}=2 \kappa v_{s}$ should be sufficiently heavy, which means that both $\lambda$ and $\kappa$ should take larger values. Finally, $y_{X}$ is an important parameter, 
which controls the chemical equilibrium decoupling temperature. We will discuss this point more detailedly later.

\section{A Sneutrino-like ADM}

In our model, the scalar components of the chiral superfieds $X$ and $\bar{X}$ (denoted by the same letters) are odd under the ordinary $R$-parity. Moreover, the dark states do not conserve a separated dark sector symmetry. Thus, the ADM candidate actually is also the LSP of the whole model. That is to say, ADM must be the lighter complex scalar from the $\left(X, \bar{X}^{*}\right)$ mixture [46]. We now explicitly check the dark sector mass spectrum. In the first, the Dirac pair approximately has a mass $M_{X}$, at the TeV scale. Secondly, $\left(X, \bar{X}^{*}\right)$ mix with the left-handed sneutrino $\widetilde{\nu}_{L}$ after electro-weak symmetry breaking. In the basis $\left(\widetilde{\nu}_{L}, X^{*}, \bar{X}\right)$, they have a mass square matrix

$$
\mathcal{M}_{S}^{2}=\left(\begin{array}{ccc}
m_{\widetilde{L}}^{2} & y_{X} A_{y_{X}} v_{u} & y_{X} M_{X} v_{u} \\
& M_{X}^{2}+m_{X}^{2} & M_{X} A_{\lambda_{X}} \\
& & M_{X}^{2}+m_{\bar{X}}^{2}
\end{array}\right) .
$$

Since the mixings with $\widetilde{\nu}_{L}$ are suppressed by $y_{X} v_{u}$, then for a heavier $m_{\widetilde{L}}^{2}$ it is justified to first diagonalize the $\left(X, \bar{X}^{*}\right)$ subsystem and get two eigenstates:

$$
X_{1}=\cos \theta_{X} X+\sin \theta_{X} \bar{X}^{*}, \quad X_{2}=-\sin \theta_{X} X+\cos \theta_{X} \bar{X}^{*},
$$

with $\theta_{X}$ the mixing angle. The corresponding eigenvalues are given by

$$
m_{1,2}^{2}=M_{X}^{2}+\frac{m_{X}^{2}+m_{\bar{X}}^{2}}{2} \mp \sqrt{\left(m_{X}^{2}-m_{\bar{X}}^{2}\right)^{2}+4\left(M_{X} A_{\lambda_{X}}\right)^{2}} .
$$

$X_{1}$, the lighter eigenstate, is the ADM (We will identify $X_{1} \mid$ vacuum $\rangle$ and $X_{1}^{\dagger} \mid$ vacuum $\rangle$ as $\mathrm{DM}$ and anti-DM, respectively). It is noticed that $m_{1}^{2}$ can be much smaller than $M_{X}^{2}$ in the presence of a large $A_{\lambda_{X}}$ or negative soft mass squares.

The mixing between $X_{1}$ and $\widetilde{\nu}_{L}$ endows ADM with sneutrino properties and hence allows it to two-body decay into a pair of leptons along the operator $L L E^{c}$. In addition to that, the mixing may matter in the later discussions on ADM, such as the ADM chemical equilibrium with neutrinos. So, we later convenience we estimate the mixing angle:

$$
C_{X \widetilde{\nu}_{L}} \simeq \frac{y_{X} v_{u}}{m_{\widetilde{L}}^{2}-m_{1}^{2}}\left(A_{y_{X}} \cos \theta_{X}-M_{X} \sin \theta_{X}\right)
$$

For $y_{X} \sim \mathcal{O}(0.1)$, it is can be readily arranged to be less than $\mathcal{O}\left(10^{-4}\right)$, say by lowering $\sin \theta_{X}$ and moreover taking a relatively small $A_{y_{X}}$. A small $C_{X \widetilde{\nu}_{L}}$ helps to alleviate the need for exceedingly small $R$-parity violations. 


\section{ADM at the earlier Universe}

At the earlier Universe, the dark sector enters thermal equilibrium with the plasma via its significant couplings to $S$. But such interactions conserve the dark number and thus do not transfer asymmetry from the visible sector to the dark sector. As mentioned before, transfer proceeds only after the $L H_{u} X$ term becomes active. It leads to the neutralino-neutrino-DM interactions

$$
\mathcal{L}_{X \nu} \supset y_{X} U_{u m} X_{1} \bar{\nu} P_{R} \chi_{m}+\text { c.c. }
$$

We have used $\widetilde{H}_{u}^{0}=\sum_{m=1}^{5} U_{u m} \chi_{m}$ with $\chi_{m}$ denoting the the NMSSM five neutralinos having Majorana masses $M_{\chi_{m}}$. There are terms proportional to $C_{X \widetilde{\nu}_{1}}$ contributing to the above interactions, but they are negligible due to $C_{X \widetilde{\nu}_{1}} \ll y_{X}$. Interactions in Eq. (12) give rise to the following scattering

$$
X_{1} \nu \leftrightarrow X_{1}^{*} \bar{\nu}
$$

which then maintains the chemical equilibrium between the dark states and the light species. The chemical potential of DM is determined to be

$$
\mu_{X}=-\mu_{\nu}
$$

$\mu_{\nu}$ expresses the lepton chemical potential and is related to the quark chemical potential. It is positive, so we have a negative $\mu_{X}$ and then only DM survives.

It is able to precisely determine the ratio $x_{D} \equiv T_{D} / m_{1}$, with $T_{D}$ the decoupling temperature at which the chemical equilibrium of the process Eq. (13) breaks. Therefore, once the ADM mass $m_{1}$ is fixed, says $m_{1}=2.39 \mathrm{TeV}$ in our model (See section II), $T_{D}$ can be determined also. To get that heavy ADM, we are expecting $x_{D} \sim \mathcal{O}(0.1)$, so it is reasonable to consider $T_{D} \gtrsim 200 \mathrm{GeV}$. It lies above the sphaleron decoupling temperature, further assuming that it is above the critical temperature of electroweak phase transition, and then we have

$$
\frac{n_{B}}{n_{X_{1}}}=\frac{8}{5 k_{X}} f_{X}^{-1}\left(m_{1} / T_{D}\right) .
$$

To get it we have decoupled the sparticles because we are considering a rather heavy SUSY spectrum. The factor $k_{X}$ depends on the dark sector mass spectrum, and its value varies between 1 and 3. We will comment on it soon later. Now, from Eq. (15) and Eq. (6) we get $x_{D} \simeq 0.08$ and $T_{D} \simeq 200 \mathrm{GeV}$ (This justifies the previous assumption on $T_{D}$ ) after taking $k_{X}=1$. Note that $x_{D}$ is not sensitive to $k_{X}$ and $m_{1}$, while $T_{D}$ has a strong dependence on $m_{1}$ but not $k_{X}$, which can be seen explicitly on Fig. 2 . Such features are traced back to the power law behavior of $f_{X}^{-1}\left(x_{D}\right)$, see Eq. (5). 


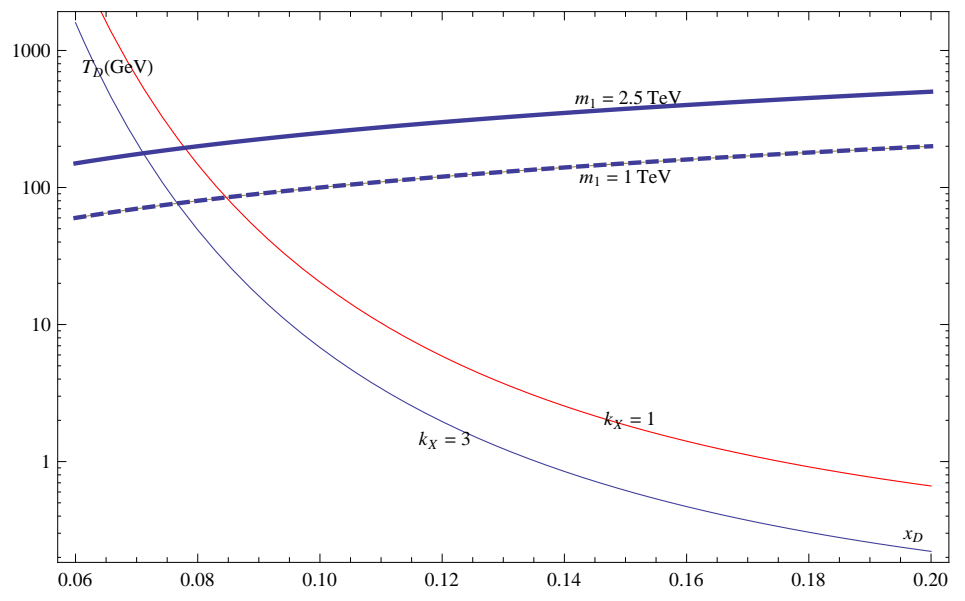

FIG. 2: A plot of heavy ADM solution to the cosmic coincidence puzzle, on the $x_{D}-T_{D}$ plane. For given ADM mass $m_{1}$ and the $k_{X}$ factor, the intersections between the two lines determine $x_{D}$ and then the chemical equilibrium decoupling temperature $T_{D}$.

We now proceed to study how $T_{D}$ arrives. As a rough estimation, $T_{D}$ can be determined by equaling $\Gamma_{X \nu}\left(T_{D}\right)$, the thermal average scattering rate of Eq. (13), to the Hubble expansion rate $H\left(T_{D}\right)=1.66 g_{*}^{1 / 2} T_{D}^{2} / M_{\mathrm{Pl}}$. Here $g_{*}$ is the effective relativistic degrees of freedom at $T_{D}$, and it is at the order of 100 . The scattering rate is estimated to be

$$
\Gamma_{X \nu}\left(T_{D}\right) \simeq n_{X}\left\langle\sigma_{X \nu} v\right\rangle \simeq\left(0.064 g_{X_{1}} m_{1}^{3} x_{D}^{-3 / 2} e^{-1 / x_{D}}\right) \frac{\left|y_{X} U_{u m}\right|^{4}}{64 \pi} \frac{M_{\chi_{m}}^{2}}{\left(M_{\chi_{m}}^{2}-m_{1}^{2}\right)^{2}} 2 x_{D},
$$

with $g_{X_{1}}=2$ the internal degrees of freedom of the complex scalar ADM. One of the Feynman diagrams for the scattering cross section has neutralinos in the $s$-channel, so it shows a resonant behavior as $M_{\chi_{m}}$ approach to $m_{1}$. To decouple the chemical equilibrium at $T_{D} \sim 200 \mathrm{GeV}, y_{X}$ can not be too small. Concretely, from the above equation we need

$$
\left|y_{X} U_{u m}\right|^{4} \frac{M_{\chi_{m}}^{2} / m_{1}^{2}}{\left(M_{\chi_{m}}^{2} / m_{1}^{2}-1\right)^{2}} \sim 10^{-9} .
$$

Thus, $y_{X} \sim 0.1$ is needed even if a moderately large resonant enhancement is provided.

Comments are in orders. First, we have neglected the scattering process $X_{1} \nu \rightarrow \widetilde{t t}$, of which the scattering cross section is large and at the order of $\mathcal{O}\left(y_{i X}^{2} y_{t}^{2}\right)$. But stops are much heavier than $X_{1}$ while at $T_{D}$ the typical kinematic energy available for $X$ and $\nu$ is only $E \sim T_{D} \sim 100 \mathrm{GeV}$, which is inadequate to maintain the chemical equilibrium of that scattering process. Consequently, it decouples at a temperature much higher than $T_{D}$ and is irrelevant to our discussion. Next, here the scattering is just the process which maintains the chemical equilibrium, and thereby the neutralino mediated wash-out effect is not of concern. Finally, other dark sector states, the Dirac pair $(\widetilde{X}, \widetilde{X})$ and $X_{2}$ store appreciable 
DM asymmetries given that their masses are near $m_{1}$. The former can establish chemical equilibrium with neutrinos via Higgs mediated scattering. To account for this uncertainty, we introduce the factor $k_{X}$ which has been used previously:

$$
k_{X}=\sum_{X_{i}} \frac{g_{X_{i}}}{g_{X_{1}}} \frac{f\left(m_{X_{i}} / T_{D}\right)}{f\left(m_{1} / T_{D}\right)},
$$

with $X_{i}$ running over $X_{1,2}$ and the Dirac pair. Clearly, we have $1 \leqslant k_{X} \leqslant 3$, with the upper bound saturated when $X_{i}$ are degenerate.

As the Universe cools down to $T_{f}$, the ADM annihilates away its symmetric component by means of DM and anti-DM collisions, which requires a moderately large annihilation

rate $\gtrsim 10 \mathrm{pb}$ [32]. For a $\mathrm{TeV}$ scale $\mathrm{DM}$, generically this is problematic if we require $\lambda_{X}$ and $\lambda$ sufficiently small so as to maintain perturbativity of the model up to the GUT scale. Fortunately, the singlet sector is heavy (For simplicity, it is assumed to decouple from the Higgs doublets), and thus the process $X_{1} X_{1}^{*} \rightarrow H_{1} H_{2}$ can be resonantly enhanced by the singlet-like CP-even Higgs bosons $H_{3}$ in the $s$-channel. Here $H_{1,2}$ are $H_{u}$ - (thus SM-) and $H_{d}$-like, respectively. In this decoupling limit, it is ready to calculate the cross section

$$
\begin{aligned}
\sigma_{H_{1} H_{2}} v & \simeq \frac{\lambda_{X}^{2}}{64 \pi}\left(\frac{M_{X}^{2}}{m_{1}^{2}}\right)\left(\frac{\left(\lambda A_{\lambda}\right)^{2}}{4 m_{1}^{4}}\right) f\left(4 m_{1}^{2} / m_{H_{3}}^{2}\right) \\
& =7.8 \times\left(\frac{\lambda_{X}^{2}}{0.1}\right)\left(\frac{\lambda A_{\lambda} M_{X}}{10 \mathrm{TeV}^{2}}\right)^{2}\left(\frac{2 \mathrm{TeV}}{m_{1}}\right)^{4} \mathrm{pb},
\end{aligned}
$$

where the Breit-Wigner enhancement factor $f$ is set to 100 . Note that a large trilinear soft term $A_{\lambda}$, which is necessary to get a large $v_{s}$, also helps to increase the above cross section. In a word, it is not difficult to get a sufficiently large cross section to annihilate away the symmetric part of $n_{X_{1}}$.

\section{ADM today}

We now show how does the ADM leptonic decay produce the desired asymmetric CR in our model. $X_{1}$ shares the couplings of left-handed sneutrinos $\widetilde{\nu}_{L, i}$, so, along the $R$-parity violating operators $L L E^{c}$, it can (only) decay into a pair of charged leptons. From the data fitting in Section II, the $\mu^{-} \tau^{+}$mode is singled out. To this end, we let $\lambda_{123} y_{X 1}$ dominate over other similar products (This may be arranged by a flavor symmetry) and then the resulted operator for ADM decay is

$$
\mathcal{L}_{\text {decay }} \supset C_{X \widetilde{\nu}_{1}} \lambda_{123} X_{1} \bar{\tau} P_{L} \mu .
$$

Note that its Hermit conjugated term is irrelevant to the ADM decay, because only DM is left today. In other words, the mode with final states $\mu^{+} \tau^{-}$is absent in the ADM decay. 
The $e^{-}$and $e^{+}$spectra respectively from $\mu^{-}$and $\tau^{+}$are different, and therefore we obtain an asymmetric $\mathrm{CR}$. In addition, the ADM life time is given by

$$
\tau\left(X_{1} \rightarrow \tau^{+} \mu^{-}\right) \approx 1.7 \times 10^{26}\left(\frac{10^{-4}}{C_{X \widetilde{\nu}_{L}}}\right)^{2}\left(\frac{10^{-22}}{\lambda_{123}}\right)^{2}\left(\frac{2 \mathrm{TeV}}{m_{1}}\right) s .
$$

When $C_{X \widetilde{\nu}_{L}} \sim 10^{-4}$ or even smaller, $\lambda_{123}$ can be obviously larger than those in the previous works [10]. In the Appendix. A we will present a model to realize the extremely small $\lambda_{123}$.

It is worthwhile to note that in our model the sneutrino-like ADM decay does not produce other significant signatures such as neutrino flux or anti-protons (But electroweak corrections on the charged particles or $\tau$ hadronic decay can still induce a correlated anti-proton signature [15]). The diffuse gamma ray from ICS and tau lepton decay is an exception. Actually, as stressed in the introduction, it is stringently constrained by the Fermi-LAT gamma ray data even in the decaying DM scenario. To estimate the surviving status of the ADM with mass $2.39 \mathrm{TeV}$ and lifetime $2.23 \times 10^{26} \mathrm{~s}$, we refer to several constraints on the lifetime of $\mathrm{DM} \rightarrow \tau^{+} \tau^{-}$, using different astrophysical objects. But the bound here is (roughly) halved because only one $\tau$ is produced from the ADM decay. Then, the current data of Fermi-LAT Galactic diffuse emission and galaxy clusters respectively give the lower bound on the ADM lifetime, $1.0 \times 10^{26} \mathrm{~s}[6]$ and $3.0 \times 10^{25} \mathrm{~s}$ [33]. Thus they do not exclude the decaying ADM, but the extragalactic gamma ray background, which gives an lower bound $1.0 \times 10^{27} \mathrm{~s}[8]$, does. This approximate result is consistent with the specific study [34]. So, to avoid this moderate exclusion, we may have to tune astrophysical parameters such that the fitted lifetime is allowed.

Finally we briefly discuss the prospect on the direct detection of the ADM $X_{1}$. The lefthanded sneutrino fraction within the ADM is negligible, which implies that the $Z$-boson mediated ADM-nucleon spin-independent scattering is undetectable. Consider the $F$-term of $S:\left|F_{S}\right|^{2}=\left|\lambda_{X} X \bar{X}+\lambda H_{u}^{0} H_{d}^{0}+\ldots\right|^{2}$, from which we get the vertex (The Higgs sector is still in the decoupling limit)

$$
-\frac{1}{\sqrt{2}} \lambda \lambda_{X} v \sin 2 \beta \sin 2 \theta_{X}\left|X_{1}\right|^{2} H_{1}
$$

The SM-like Higgs boson $H_{1}=h$ may lead to a detectable spin-independent scattering cross section, which can be written as $\sigma_{p}=f_{p}^{2} \mu_{p}^{2} / \pi$ [38] with $\mu_{p} \approx m_{p}$ the reduced DM-nucleon mass. Specified to this model, we have [39]

$$
\begin{aligned}
f_{p} & =-\lambda \lambda_{X} \sin 2 \beta \sin 2 \theta_{X} \frac{m_{n}}{2 m_{1}} \frac{1}{m_{h}^{2}}\left(\sum_{q=u, d, s} f_{T_{q}}^{(p)}+3 \times \frac{2}{27} f_{T_{G}}^{(p)}\right) \\
& \approx 0.8 \times 10^{-9} \times\left(\frac{\lambda \lambda_{X}}{0.5}\right)\left(\frac{\sin 2 \beta}{0.4}\right)\left(\frac{\sin 2 \theta_{X}}{1.0}\right)\left(\frac{2 \mathrm{TeV}}{m_{1}}\right) \mathrm{GeV}^{-2}
\end{aligned}
$$


with $m_{h}=125 \mathrm{GeV}$ fixed and values of $f_{T_{u}}^{(p)}$, etc., given in Ref. [39]. Even for $\sigma_{p}$ from the above parameterization to maximize the scattering rate, we have $\sigma_{p} \simeq 0.8 \times 10^{-10} \mathrm{pb}$. On the other hand, for a TeV DM the latest XENON100 data [40] gives the most stringent upper bound $\sim 10^{-8} \mathrm{pb}$, which is far above the estimated $\sigma_{p}$. Thus, $X_{1}$ is hard to be detected, even at the next around of XENON.

\section{CONCLUSIONS AND DISCUSSIONS}

The first result of AMS-02 reported the excess of cosmic positron fraction. This confirms the previous conclusion by PAMELA, but its spectrum is relatively softer which leads to a tension with the Fermi-LAT $e^{+}$and $e^{-}$total flux spectrum measurement. We may need a somewhat unconventional extra source of CR to resolve the tension. In this article we propose a solution using the asymmetric CR from asymmetric dark matter late decay. We find that a multi-TeV ADM (about $2.4 \mathrm{TeV}$ ) asymmetrically decays into $\mu^{-} \tau^{+}$can indeed significantly improve the fit which adopts the symmetric DM decay. Based on the NMSSM with $R$-parity-violating SUSY, we construct a minimal renomalizable supersymmetric model to realize that scenario. The model only introduces a pair of singlets and involves no new scales. But the ADM is difficult to be directly detected.

In the decaying ADM framework to explain the AMS-02 and Fermi-LAT anomalies, there are some open questions needed to be answered. First, at the tree-body decay level, it is of interest to consider modes containing quarks, says $X \rightarrow e^{-} u \bar{d}$. After hadronization, we will have an asymmetric proton spectrum, and $\bar{p} /(p+\bar{p})<0.5$. Can it lead to an acceptable anti-proton spectrum by PAMELA? Next, it is not clear whether or not the ADM with multi decay modes with proper branching ratios can help to further improve the fits. Last but not the least, distinguishing the asymmetric CR generated by the ADM mechanism from the one by astrophysical mechanisms is very important. For example, it may be done by measuring the $e^{-}$spectrum precisely.

To end up this article, we would like to comment on constraints on the non-annihilating bosonic ADM. From some astrophysical objects such as the neutron star, it is can be constrained or even excluded for the light bosonic ADM [42] (but with exception [43]). However, heavy bosnic ADM has not been constrained yet [41].

\section{Acknowledgement}

We thank useful discussions with Yizhong Fan. This research was supported in part by the China Postdoctoral Science Foundation (No. 2012M521136 and 2013M530006). 


\section{Appendix A: Generating small $R$-parity violation}

The decaying ADM life time is extremely long, $\sim 10^{26} \mathrm{~s}$, and thus how to generate the extremely small $R$-parity violations is challenging. In this appendix we present a simple model to explain its smallness. To that end, we introduce a pair of vector-like lepton doublets $\left(L_{V}, \bar{L}_{V}\right)$ with mass around the Planck scale. They mediate lepton number violations, which are induced by the $F$-term of some singlet $\mathcal{X}$ (namely $F_{\mathcal{X}} \neq 0$ ) to ordinary leptons. The model is given by

$$
\frac{c_{j}}{M_{\mathrm{Pl}}} \int d^{4} \theta \bar{L}_{V} L_{j} \mathcal{X}^{\dagger}+\int d^{2} \theta \lambda_{i k} L_{i} L_{V} E_{k}^{c}+M_{V} L_{V} \bar{L}_{V}
$$

Actually, the first term generates a tiny mixing between the vector-like leptons and the light $\mathrm{SM}$ leptons. The mixing is suppressed by $F_{\mathcal{X}} / M_{V} M_{\mathrm{Pl}}$, and as a consequence the induced coefficients for the $R$-parity-violating operators $L_{i} L_{j} E_{k}^{c}$ are estimated as

$$
\lambda_{i j k} \sim \lambda_{i k} c_{j} \frac{F_{\mathcal{X}}}{M_{V} M_{\mathrm{Pl}}}
$$

It is interesting to note that $\lambda_{i j k}$ as small as $10^{-20}$ can be achieved even if $F_{\mathcal{X}} / M_{\mathrm{Pl}} \sim m_{\widetilde{G}}$ with the gravitino mass $m_{\widetilde{G}} \sim 1 \mathrm{TeV}$, the typical mass scale of soft terms in the gravitymediated SUSY-breaking. In other words, $\mathcal{X}$ may originate in the hidden sector breaking SUSY and, tampingly, is identified with the hidden sector SUSY-breaking spurion field.

[1] Planck Collaboration, P. Ade et. al., arXiv:1303.5062.

[2] O. Adriani et al. [PAMELA Collaboration], Nature 458, 607 (2009).

[3] O. Adriani, et al., Astropart. Phys. 34, 1 (2010); M. Ackermann et al. [Fermi LAT Collaboration], Phys. Rev. Lett. 108, 011103 (2012).

[4] The Fermi LAT Collaboration, A. A. Abdo et. al., Phys. Rev. Lett. 102 (2009) 181101.

[5] O. Adriani et al. [PAMELA Collaboration], Phys. Rev. Lett. 105, 121101 (2010).

[6] T. F. -: M. Ackermann et al. [LAT Collaboration], Astrophys. J. 761, 91 (2012).

[7] P. Meade, M. Papucci, A. Strumia and T. Volansky, Nucl. Phys. B 831, 178 (2010);

[8] M. Cirelli, E. Moulin, P. Panci, P. D. Serpico and A. Viana, Phys. Rev. D 86, 083506 (2012).

[9] J. Chang, et al., Nature 456, 362 (2008); F. Aharonian, et al., Phys. Rev. Lett. 101, 261104 (2008), 0811.3894.

[10] P. -f. Yin, Q. Yuan, J. Liu, J. Zhang, X. -j. Bi and S. -h. Zhu, Phys. Rev. D 79, 023512 (2009); A. Ibarra, D. Tran and C. Weniger, JCAP 1001, 009 (2010); K. Ishiwata, S. Matsumoto and T. Moroi, Phys. Lett. B 675, 446 (2009); S. -L. Chen, R. N. Mohapatra, S. Nussinov and 
Y. Zhang, Phys. Lett. B 677, 311 (2009); R. C. Cotta, et al., JHEP 1101, 064 (2011); M. Ibe, S. Iwamoto, S. Matsumoto, T. Moroi and N. Yokozaki, arXiv:1304.1483.

[11] A. Arvanitaki et al., Phys. Rev. D 79 (2009) 105022; M. Luo, L. Wang, W. Wu and G. Zhu, Phys. Lett. B 688, 216 (2010).

[12] X. Gao, Z. Kang and T. Li, Eur. Phys. J. C 69 (2010) 467; C. D. Carone and R. Primulando, Phys. Rev. D 84, 035002 (2011); Y. Kajiyama, H. Okada and T. Toma, arXiv:1304.2680.

[13] M. Aguilar, et al., Physical Review Letters 110, 141102 (2013).

[14] L. Feng, R. -Z. Yang, H. -N. He, T. -K. Dong, Y. -Z. Fan and J. Chang, arXiv:1303.0530.

[15] A. De Simone, A. Riotto and W. Xue, arXiv:1304.1336 [hep-ph].

[16] Q. Yuan, X.-J. Bi, G.-M. Chen, Y.-Q. Guo, S.-J. Lin, et al. (2013), 1304.1482.

[17] H. -B. Jin, Y. -L. Wu and Y. -F. Zhou, arXiv:1304.1997 [hep-ph].

[18] T. Linden and S. Profumo, arXiv:1304.1791; P. -F. Yin, Z. -H. Yu, Q. Yuan and X. -J. Bi, arXiv:1304.4128 [astro-ph.HE].

[19] I. Cholis and D. Hooper, arXiv:1304.1840;

[20] Q. Yuan and X. -J. Bi, arXiv:1304.2687 [astro-ph.HE].

[21] M. T. Frandsen, I. Masina and F. Sannino, Phys. Rev. D 83, 127301 (2011); I. Masina and F. Sannino, JCAP 1109 (2011) 021.

[22] S. Chang and L. Goodenough, Phys. Rev. D 84, 023524 (2011) [arXiv:1105.3976 [hep-ph]].

[23] A. W. Strong, and I. V. Moskalenko, Astrophys. J. 509212 (1998).

[24] J. F. Navarro et al., Mon. Not. Roy. Astron. Soc. 402 (2010) 21

[25] http://cosmologist.info/cosmomc/

[26] I. Masina and F. Sannino, arXiv:1304.2800 [hep-ph].

[27] Z. Kang and T. Li, JHEP 1210, 150 (2012).

[28] See a review: H. Davoudiasl and R. N. Mohapatra, New J. Phys. 14, 095011 (2012).

[29] J.A. Harvey and M.S. Turner, Phys. Rev. D 42 (1990) 3344.

[30] E. W. Kolb and M. S. Turner, The Early Universe, Addison-Wesley (1990).

[31] M. R. Buckley and L. Randall, JHEP 1109, 009 (2011).

[32] M. L. Graesser, I. M. Shoemaker and L. Vecchi, JHEP 1110, 110 (2011).

[33] Xiaoyuan Huang, et al., JCAP 1201 (2012) 042.

[34] I. Masina, P. Panci and F. Sannino, JCAP 1212, 002 (2012).

[35] P. -H. Gu, U. Sarkar and X. Zhang, Phys. Rev. D 80, 076003 (2009); P.-H. Gu, M. Lindner, U. Sarkar and X. Zhang, Phys. Rev. D 83 (2011) 055008; Y. Cui, L. Randall and B. Shuve, JHEP 1204, 075 (2012); S. Davidson and M. Elmer, JHEP 1210, 148 (2012); H. Kuismanen and I. Vilja, Phys. Rev. D 87, 015005 (2013).

[36] D. E. Kaplan, M. A. Luty and K. M. Zurek, Phys. Rev. D 79, 115016 (2009) 
[37] Z. Kang, J. Li, T. Li, T. Liu and J. Yang, arXiv:1102.5644 [hep-ph].

[38] G. Jungman, M. Kamionkowski and K. Griest, Phys. Rept. 267, 195 (1996).

[39] X. Gao, Z. Kang and T. Li, JCAP 1301, 021 (2013).

[40] E. Aprile et al. [XENON100 Collaboration], Phys. Rev. Lett. 109, 181301 (2012).

[41] C. Kouvaris and P. Tinyakov, arXiv:1212.4075 [astro-ph.HE].

[42] S. D. McDermott, H.-B. Yu and K.M. Zurek, Phys. Rev. D 85 (2012) 023519; C. Kouvaris and P. Tinyakov, Phys. Rev. Lett. 107 (2011) 091301; I. Lopes and J. Silk, Astrophys. J. 757, 130 (2012);

[43] N. F. Bell, A. Melatos and K. Petraki, arXiv:1301.6811.

[44] ATIC and HESS [9], etc., also reported total flux excess, but for simplicity we only mention Fermi-LAT hereafter.

[45] A similar point was taken in the recent work [26] which appeared on the arXiv during the preparation of this work. Ref. [17] also considered the asymmetric CR scenario but drew a negative conclusion. Basically, that may be due to the fact that authors of Ref. [17] actually took a constant instead of energy-dependent asymmetry.

[46] Within the MSSM, the left-handed sneutrino in principle can be a leptonic decaying ADM proposed in the text. This is achieved by suppressing the neutralino mediated charge wash-out effect via a very heavy neutralino spectrum, says the bino has a mass around $100 \mathrm{TeV}$. We leave such an interesting scenario for future study. 